\title{
ANÁLISIS DE MANUALES A TRAVÉS DEL TRATAMIENTO DIDÁCTICO DADO AL CONCEPTO DE LÍMITE DE UNA FUNCIÓN: UNA PERSPECTIVA DESDE LA NOCIÓN DE OBSTÁCULO
}

\author{
SÁNCHEZ GOMEZ, C. ${ }^{1}$ y CONTRERAS DE LA FUENTE, A. $^{2}$ \\ I Área de Análisis Matemático. Universidad de Jaén. \\ ${ }^{2}$ Área de Didáctica de la Matemática. Universidad de Jaén.
}

\section{SUMMARY}

This paper is based on Sierpinska's ideas (1991) about the notions of comprehension acts and epistemological obstacles, and on other basic concepts. Therefore, in this study we try to obtain some methodological implications related to the teaching of the functional limit notion, through the analysis of some textbooks. The following variables are taken into account: $a$ ) the way the concept is presented; $b$ ) the type of definition; $c$ ) the examples appearing in the previous sections or headings; $d$ ) the conceptions of limit which can be derived from the textbook; $e$ ) possible obstacles and difficulties in the development of the contents, our attention being mainly centered on conceptions and obstacles.

Student: The car has a speed of 50 miles an hour. What does that mean? Teacher: Given any $\varepsilon>0$, there exists a $\delta>0$ such that if $\left|t_{2}-t_{1}\right|<\delta$, then

$$
\frac{s_{2}-s_{i}}{t_{2}-t_{i}}-50<\varepsilon
$$

Student: How in the world did anybody ever think of such an answer? (Judith V. Grabiner. American Mathematical Monthiy, 1983.)

\section{INTRODUCCIÓN}

Una de Ias nociones fundamentales del análisis matemático es la de límite de una función, tanto por su carácter operativo, en la definición de otros conceptos básicos y en la resolución de numerosos problemas en la propia matemática o en otras ciencias (por ejemplo, física e ingenierías) como por su carácter de objeto matemático teórico en sí mismo. Sin embargo, su enseñanza presenta múltiples problemas relacionados, por una parte, con las diversas concepciones del concepto (Delediq, 1994; Sánchez y Contreras, 1995a) y, por otra, con los obstá- 
culos cognitivos que jalonan el mismo (Cornu, 1983, 1985; Sierpinska, 1991; Sánchez y Contreras, 1995b).

Son numerosos los estudiantes que, por una realización no adecuada de la transposición didáctica efectuada desde el saber enseñar al saber escolar (ver diagrama siguiente), que es precisamente donde intervienen los programas y los manuales, se encuentran con problemas cuando pretenden adquirir o mejorar sus conocimientos a través de los textos, detectándose dificultades y obstáculos por medio de sus errores. En este sentido, Rico (1992) afirma: «Siendo un objetivo permanente de Ia enseñanza de las matemáticas en el sistema escolar lograr un correcto aprendizaje de las mismas por parte de Ios alumnos, es claro que las producciones o respuestas incorrectas a las cuestiones que se les plantean se consideran como señales de serias deficiencias e incluso fracaso en el logro de dicho objetivo.»

Uno de Ios objetivos que se pretende lograr con este trabajo es extraer conclusiones, a través de los datos obtenidos, que permitan aportar orientaciones sobre lo que se debería tener en cuenta en la elaboración de textos para el estudiante, facilitando de esta manera la comprensión del concepto. En la primera parte, se hace una breve descripción de las concepciones y de los obstáculos sobre la noción de límite de una función. Posteriormente se presentan los restultados de un estudio de diversos manuales de matemáticas de enseñanza no universitaria y del primer curso de universidad, realizado según las ideas de Schubring (1985, 1987), Weber (1986) y Romero (1991), en el que se señalan, entre las variables observadas, Ias concepciones seguidas por los autores en el desarrollo del concepto, así como los obstáculos epistemológicos y didácticos a los que los textos pueden inducir, y, por último, se indican algunas conclusiones. El arł́culo es parte de un estudio más amplio sobre la noción de limite de una función (Sánchez y Contreras, 1996a), del que se espera extraer conclusiones que aporten información a los profesores sobre la enseñanza de dicha noción.

\section{LOS MANUALES Y EL SABER ESCOLAR}

El conjunto de adaptaciones que experimenta un determinado concepto matemático, desde su condición de saber científico (conjunto de conocimientos que han sido objeto de publicaciones científicas y reconocidos como válidos por toda una comunidad) hasta la de saber escolar (conjunto de conocimientos que aparece en los programas y manuales), es lo que se denomina transposición didáctica (Chevallard, 1991). En sentido amplio, se trata de un proceso con un desarrollo más complejo en el que intervienen múltiples aspectos: en primer lugar, el profesor ha de adaptar sus propios conocimientos acerca de los objetos que se deben enseñar, a continuación debe insertarlos en el saber escolar y, por último, organizarlos temporalmente; es decir, debe realizar una transición del saber escolar al saber enseñado, teniendo en cuenta que este último nunca es totalmente retenido por el estudiante, sino que es a través de la relación didáctica que se establece entre el profesor y los alumnos, como el saber enseñado se convierte en saber del alumno.

Todo lo anterior se describe en el esquema I simplificado, adaptado de Henry (1991).

Cuando en los manuales se realiza el paso del saber que debe ser enseñado al saber escolar, se manifiestan, entre otros, concepciones, obstáculos (epistemológicos y didácticos) y también dif́cultades; todos ellos relacionados con el saber, cuyo análisis nos puede permitir extraer consecuencias pertinentes acerca de la enseñanza de los conceptos matemáticos. Es a través de las interacciones que se producen entre el saber, el profesor y los estudiantes como éstos construyen progresivamente sus conocimientos pasando por concepciones sucesivas; es decir, la comprensión de un concepto trae consigo una nueva forma de conocimiento que, en sentido epistemológico, corresponde al término concepción, y que designa, por tanto, la forma en que un sujeto comprende y utiliza un concepto. Dado que se trata de un análisis de manuales, estamos interesados en aquellas concepciones que se

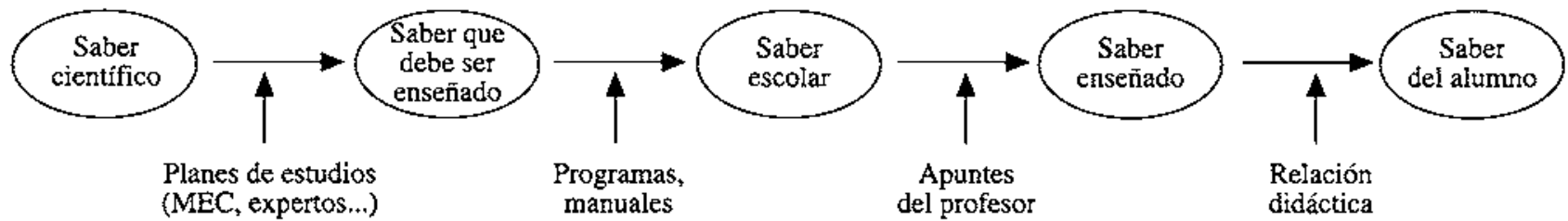


identifican en la génesis histórica de una noción matemática; son concepciones históricas (El Bouazzoui, 1988), identificadas en una comunidad de matemáticos y, por tanto, colectivas. Uno de nuestros objetivos es precisamente establecer el tipo de concepción histórica que se manifiesta en los manuales que se analizan.

Por otra parte, de acuerdo con El Botlazzoui (1988), la evolución de todo concepto matemático hace que la elaboración del mismo sea un proceso en el que aparecen dificultades y obstáculos. La dificultad se presenta cuando la solución del problema que surge en un determinado momento de enriquecimiento estructural del concepto no precisa un cambio básico de la teoría matemática correspondiente. Los obstáculos se caracterizan por las cinco condiciones siguientes:

1) Es un conocimiento y no una ausencia del mismo.

2) Permite dar respuesta a cierto tipo de problemas.

3) Conduce a respuestas erróneas en otro tipo de problemas.

4) Es resistente a toda transformación o modificación y se manifiesta de forma recurrente.

5) Su superación conduce a un conocimiento nuevo.

Es decir, aparecen cuando la solución del problema que surge en un determinado momento de enriquecimiento estructural del concepto precisa de un cambio importante en la teoría; son, por tanto, inherentes a la génesis del concepto y Bachelard (1983) los denomina obstáculos epistemológicos. Obviamente, los obstáculos tienen una gran relevancia para la construcción del saber en cada individuo una vez que son superados.

Sierpinska (1991), al realizar un estudio epistemológico del concepto đe límite de una sucesión, señala que un acto de comprensión de un concepto es un fenómeno psíquico cuyo objetivo es apropiarse de los significados relativos a elementos particulares de aquél. Distingue cuatro actos de comprensión: identificación (de objetos que pertenecen a la denotación del concepto), discriminación (entre dos objetos que antes estaban confusos), generalización (ser consciente de la no-esencialidad de algún supuesto, o de la posibilidad de ampliar el rango de aplicaciones) y sintetización (captar la relación entre dos o más objetos y organizarlos dentro de un todo consistente). Esta autora afirma que algunos actos de comprensión, lo son de superación de obstáculos, por lo que la comprensión de un concepto matemático consistiría en la superación, a través de los actos de comprensión pertinentes, de un conjunto de obstáculos relativos al concepto que proporcionarian una amplia información sobre su significado; señala, además, que la comprension de un concepto se podría medir por el número y la calidad de los obstáculos epistemológicos relativos a él y que uno haya superado. Al estudiar los manuales $y$, posteriormente, reflejar los resultados en tablas, se ha considerado que, si un determinado manual induce con su exposición a un obstáculo y no se plantean situaciones que provoquen actos de comprensión para la superación del mismo, dicho obstáculo permanecerá en la tabla, incluyéndose en obstáculos inducidos (OI) y en obstáculos cuya superación no facilita el manual (ONS). Por otra parte, en los manuales también se han identificado obstáculos didácticos, inherentes a la necesidad de la transposición didáctica y creados por la elección de una determinada estrategia de enseñanza, los cuales conducen, durante el aprendizaje, a conocimientos erróneos o incompletos que se revelan posteriormente como obstáculos en el desarrollo de la conceptualización. En este tipo de obstáculos se ha seguido la misma metodología de análisis que en el caso de los epistemológicos.

\section{CONCEPCIONES Y OBSTÁCULOS LIGA- DOS A LA NOCIÓN DE LIMITE DE UNA FUNCION}

A efectos del análisis de los manuales que se describe aquí, se distinguen varias concepciones sobre el concepto de límite ligadas al uso de los dominios donde se desarrollan las actividades observadas. Siguiendo Ias ideas de El Bouazzaoui (1988), Cornu (1983) y Deledicq (1994) y según se desarrolla en Sánchez y Contreras (1995a), se distinguen las concepciones históricas siguientes:

CG: Concepción geométrica, relacionada con situaciones ligadas al contexto geométrico, como, por ejemplo, la aproximación đe las áreas de polígonos inscritos en un círculo según se aumenta el número de lados.

CN: Concepción numerica, ligada a la utilización de sucesiones de valores de la variable independiente y las correspondientes de la variable dependiente.

CAM: Concepción analítica o métrica, relacionada con la introducción de las variables Jógicas $\varepsilon$ y $\delta$.

CT: Concepción topológica, que es la definición más general y en la que se utiliza el concepto de punto de acumulación.

Para la determinación de los obstáculos, tanto epistemológicos como didácticos, que se describen a continuación, así como para la descripción de algún acto de comprensión necesario para su superación, se han tenido en cuenta las consideraciones anteriores y aportaciones de otros investigadores. El Bouazzaoui (1988) señala que «el límite de una función en un punto $x_{0}$ se presenta primeramente considerando una función definida sobre un intervalo de centro $x_{0}$, salvo en $x_{0}$; el límite de la función en este punto se explica como aquél que se obtiene cuando se aproxima a un lado y otro de $x_{0}$. Esta decisión de particularizar así la noción se ha impuesto por ciertas razones, entre otras, para facilitar Ia comprensión de los alumnos.» (pp. 22-23). Sin embargo, esta concepción origina interpretaciones en los alumnos que les obstaculiza posteriormente la comprensión topológica de límite.

Cornu (1983) enumera, entre los obstáculos inherentes a Ia noción de limite y observados a lo largo de la historia, los siguientes: 1) el «aspecto metafísico» đe la noción, ligado con el infinito, ya que introduce una nueva forma 
de razonamiento; 2) la «transposición numérica», obstáculo que se presenta al intentar aritmetizar la noción; 3) los conceptos de cantidades infinitamente grandes y cantidades infinitamente pequeñas, por su propia naturaleza; 4) «¿el límite... se alcanza o no?», señalando que la palabra límite puede abarcar realidades muy distintas.

Sierpinska (1985) propone una clasificación de los obstáculos inherentes a la noción de límite, que, señala que no es definitiva ni exhaustiva, en los cinco grupos siguientes: 1) obstáculos debidos al horror infiniti, 2) obstáculos ligados a la noción de función, 3) obstáculos geométricos, 4) obstáculos lógicos, 5) obstáculo del símbolo. Por último, Sierpinska (1991) realiza un estudio respecto de los obstáculos y actos de comprensión relativos a la noción de límite de una sucesión cuyo análisis, adaptación y ampliación para el límite de una función constituyen una parte de este trabajo. Por todo lo anterior, se destacan como relevantes en la investigación los siguientes obstáculos:

$\mathrm{O}_{1}$ : Centrarse en la forma de las aproximaciones de los valores de las variables independiente y dependiente más que en las propias aproximaciones.

Este obstáculo, inherente a la noción de límite, origina que los estudiantes, al calcular el límite de una función $f$ en el punto $x=2$ cuyas aproximaciones numéricas son 0,$39 ; 0,0399 ; 0,003999 ; . .$. y 0,$41 ; 0,0401 ; 0,004001 ; .$. , crean que dicho límite es 0,004 . O también, al calcular

$$
\lim _{x \rightarrow 1} \frac{2 x}{3}
$$

crean que los valores 0,$6 ; 0,66 ; 0,666 \ldots$ se aptoximan a 6 en vez de a $2 / 3$. O bien, para los valores: 0,$9 ; 0,99$; $0,999 \ldots$ que se aproximan a 9 en vez de a 1 . La discriminación entre número y forma de número, tanto para la variable dependiente como independiente permitiría la superación de este obstácưio.

$\mathrm{O}_{2}:$ Uso exclusivo de la aproximación gráfica sin acom. pañarse de los correspondientes valores de las aproximaciones numéricas de las variables independiente y dependiente.

Este obstáculo, de origen didáctico, conduce al alumno a un camino sin salida cuando no dispone de una representación gráfica, al no poder razonar sin el apoyo de la misma (en la mayoría de los manuales aparece sólo la gráfica de una función abstracta para la interpretación geométrica del límite). Este hecho junto a lo indicado por Sierpinska (1985), respecto a la forma ingenua en la que los alumnos utilizan las calculadoras y a que la tarea de realizar cálculos aproximados está muy descuidada en la enseñanza, nos hace señalar que serían actos de comprensión necesarios para superar este obstáculo el uso simultáneo de la tabla de valores y la gráfica de una función, la identificación de la tabla de valores de las aproximaciones numéricas con la gráfica correspondiente, así como discriminar de entre varias gráficas cuál es la relativa a la función cuyo límite se calcula y del que conocen las aproximaciones numéricas.

$\mathrm{O}_{3}$ : Creer que la existencia de límite de una función en un punto significa que los valores de la variable dependiente se acercan a algo.

Este obstáculo, de carácter epistemológico, está presente cuando el alumno no distingue entre aproximación y distancia y, por ello, discriminar entre aproximarse los valores de la variable dependiente al límite y aproximarse «tanto como queramos» a él es un acto de comprensión que permite superarlo. También se presenta este obstáculo cuando se considera la gráfica de una función constante, pues, al no detectar una aproximación en el sentido físico de movimiento, los alumnos dicen que no hay límite.

$\mathrm{O}_{4}:$ Creer que existe limite de una función en un punto cuando el número de valores de la variable dependiente que se acercan a él es infinito (o muy grande) y no casi todos ellos.

Esto da lugar a casos donde el alumno cree que una función que sólo tiene un límite lateral, tiene límite en el punto. Por ello, discriminar entre «un número infinito de términos se aproximan al límite» y «casi todos Ios términos se aproximan al límite» sería un acto de comprensión necesario para superar este obstáculo epistemológico.

\section{$\mathrm{O}_{5}:$ Creer que el entorno es siempre simétrico.}

Este obstáculo está ligado a la transposición didáctica que se hace de la noción de límite a través de la concepción analítica (CAM) y la concepción topológica (CT). El alumno cree que el entorno abierto ha de ser siempre simétrico, hecho que le crea errores cuando considera $f(x)=2 x$ si $x<1$ y $f(x)=x+1$ si $x>1$ y calcula el límite en $x=1$. La discriminación entre la simetría de la gráfica de la función y la amplitud del entorno del punto en el que se calcula el límite permitiría superar este obstáculo.

\section{$\mathrm{O}_{6}$ : Creer que las variables independiente o dependiente toman el valor de $\infty$.}

Éste es el origen de que algunos estudiantes consideren las expresiones:

$$
\frac{I}{\infty}, \infty, \frac{1}{0}
$$

como números. Discriminar entre número y conceptos tales como cantidades infinitamente grandes e infinitamente pequeñas sería un acto de comprensión para este obstáculo, que en nuestra opinión tiene un doble carácter; por una parte, puede considerarse de origen didácti$c o$, ya que, a veces, esta situación está inducida por el profesor o por algún manuai al utilizar una notación inadecuada cuando, al dar la definición de límite finito en un punto añaden que $a$ y $L$ pueden ser $+\infty$ y $-\infty$; por 
otra, debido a la inclusión del propio $\infty$ y al obstáculo 4 señalado por Cornu (1983), se puede considerar también epistemológico.

$\mathrm{O}_{7}$ : Creer que las letras $\varepsilon$ y $\delta$ que aparecen en la definición formal de limite de una función en un punto representan magnitudes constantes $o$ variables.

Este obstáculo, inherente a la noción, está referido al lenguaje simbólico y aparece ligado a la comprensión formal de límite de una función. Un acto de comprensión sería discriminar entre el uso de las letras en álgebra y en lógica; y según Tall (1995), como al decir que para cualquier $\varepsilon>0$ existe un $\delta>0 \ldots$, el alumno podría pensar que para todo valor de $\varepsilon$ posible existe sólo ese $\delta$, una lectura de la definición en la forma: Para «cada» $\varepsilon>0$, cualquiera que sea, existe $\delta>0 \ldots$ para «ese» $\varepsilon>0$, le ayudaría a superarlo. Además, Sierpinska (1985) señala que no basta con conocer los cuantificadores y sus propiedades para darse cuenta del papel que su presencia y su orden juegan en la definición de la noción de límite, pudiendo hacerse más claro este papel en los ejemplos de inexistencia de límite.

$\mathrm{O}_{8}$ : Creer que existe límite de una función en un punto porque la diferencia entre los sucesivos valores de la variable dependiente va disminuyendo.

Este obstáculo, propio de la noción, hace que al confundir convergencia con disminución de una distancia, para la función $f(x)=1$, si $x$ es irracional y $f(x)=x$, si $x$ es racional, Ios alumnos afirmen que hay límite en el punto $x=2$ porque observan que la diferencia entre las imágenes, para $x$ racional, se va haciendo cada vez más pequeña (identifican con sucesiones de Cauchy), por lo que la discriminación entre sucesión convergente y sucesión de Cauchy, en Ios distintos conjuntos numéricos, sería uno de los actos de comprensión necesarios.

$\mathrm{O}_{9}$ : Creer que el límite de una función en un punto es el valor de la función en ese punto.

Se favorece este obstáculo, de caracter didáctico, cuando se acude a ejemplos con funciones como la función $f$ dada por

$$
f(x)=\frac{x^{2}-4}{x-2}
$$

en Ios que se pide el límite en $x=2, y$ se intenta explicar al alumno que, salvo en 2 , la función $f(x)=g(x)=x+2$. La discriminación entre imagen de una función en un punto y valor aproximado, distinguir entre función y conjunto de sus valores, asf́ como evitar el uso de ejemplos como el anterior, permitiría superarlo.

\section{ANÁLISIS DE MANUALES}

Una de las causas que motivaron la realizacion de este trabajo fue observar que el tratamiento que se reflejaba en los manuales sobre la noción de límite de una función experimentaba variaciones metodológicas en el transcurso del tiempo. Este hecho originó la revisión de algunos manuales de los niveles de enseñanza media y universitaria (Matemáticas de $2^{\circ}$ de BUP, Matemáticas I de COU y primer curso de diplomaturas de escuelas técnicas), a fin de detectar esas variaciones, estudiar sus causas y las posibles consecuencias sobre la enseñanza. En el nivel de enseñanza media se analizan: el curso de $2^{\circ}$ de BUP (por ser en éste donde se inicia el estudio de la noción de límite de una función) y el curso de orientación universitaria (debido a que en éste se repasa y se amplía el concepto). En enseñanza universitaria se han tenido en cuenta manuales generales enfocados, sobre todo, a los primeros cursos para escuelas técnicas y, en algún caso, para facultades de ciencias. En ambos casos se han clasificado los manuales por bloques, realizándose un análisis comparativo por niveles dentro de cada uno de ellos. En este trabajo se presenta el estudio de diez manuales y, debido a la clasificación realizada, uno de ellos se ha incluido en dos bloques distintos. Dichos manuales no son los únicos existentes en cada período, para su elección se ha tenido en cuenta su difusión nacional y relevancia en la época, y se ha optado por reseñarlos como manual número ; no obstante, quedan claramente identificados al final del trabajo.

A la hora de diseñar el análisis se ha considerado, en un caso, el estudio de un mismo autor en distinto nivel de enscñanza o en distinto perf́odo y, en otro, el de distinto autor en un mismo período, optando, por tanto, por un equilibrio entre las ideas presentadas por Schubring (1985, 1987) y las presentadas por Weber (1986). Además, es importante señalar que, para el análisis, se ha considerado el momento en que el concepto de límite de una función aparece por primera vez en cada manual, pues este hecho es determinante para el futuro desarrollo de la noción e indica el enfoque didáctico con el que es tratado (como recurso didáctico en la enseñanza de otros conceptos en matemáticas o como objeto matemático de enseñanza). Para cada manual se han estudiado las variables:

1) Forma de introducir la noción de límite (con ejemplo, con definición, antes o después de límites laterales, tras continuidad).

2) Definición con la que se formaliza la noción.

3) Ejemplos que aparecen en los apartados anteriores.

4) Concepciones que se derivan del manual.

5) Obstáculos y dificultades que se extraen de los contenidos (señalando en las tablas para los obstáculos inducidos [OI] y para los obstáculos cuya superación no facilita el manual [ONS]).

Según Schubring $(1985,1987)$, es interesante la elección de un solo atltor y el estudio de las distintas ediciones, lo que permite observar los cambios en las mismas. Por tanto, se han clasificado por períodos, niveles y autores, distinguiéndose cuatro bloques:

Bloque I: Periodo: 1973-74; Niveles: E. Media (COU) $1^{\circ}$ Universidad; Características: Igual período; distintos niveles; el mismo autor. 
Bloque II: Periodo: 1983-87 / 1990-93; Niveles: E. Media ( $2^{\circ}$ de BUP y COU); Características: Distintos períodos; igual nivel; los mismos autores.

Bloque III: Período: 1987-89/ 1992; Nivel: E. Media ( $2^{\circ}$ de BUP, COU) y $1^{\circ}$ Universidad. Características: Distintos períodos; distintos niveles; el mismo autor.

Bloque IV: Periodo: 1992; Nivel: $1^{\circ}$ Universidad; Caracteristicas: Igual período; igual nivel; distinto autor.

En cada uno de los bloques se ha realizado un análisis pormenorizado de las variables indicadas; las dimensiones de este artículo no permiten transcribir todo el estudio, por lo que únicamente se reflejan, en el análisis de cada bloque, algunos de los aspectos más significativos correspondientes, sobre todo, a las concepciones y obstáculos. El estudio de las demás variables se ha restumido en tablas.

\section{Análisis del bloque I (Resultados en tabla I)}

En el manual 1 aparece reiteradamente el término suficientemente próximo, pero, al no incluir ninguna discusión sobre el término aproximación, no se facilita que el alumno realice los actos de comprensión que le permitan superar este obstáculo, por lo que se mantiene el obstáculo $\mathrm{O}_{3}$. Debido a la forma de introducir el concepto, a la definición y ejemplo que se utiliza, se derivan las concepciones numérica (CN) y analítica-métrica (CAM). En la transposición didáctica que se realiza, el autor hace uso de la gráfica de una función abstracta (un trazado cualquiera que representa una supuesta función) con el objetivo de lograr una mejor comprensión del concepto; sin embargo, este hecho induce al obstáculo $\mathrm{O}_{2}$, ya que, al no relacionarse la gráfica que se pone de ejemplo con una tabla de valores con las aproximaciones numéricas, no se favorecen los actos de comprensión necesarios para superarlo. Respecto al manual 2 se mantienen las mismas concepciones y obstáculos que en el manual 1, ya que el autor da idéntico tratamiento al concepto, y sólo diferencia los dos niveles de enseñanza para los que están destinados dichos textos, con la inclusión de tres ejemplos, una vez que define la noción de límite finito de una función en un punto. En cuanto a las concepciones, es de destacar la ausencia en el manual universitario de la noción de punto de acumulación, hecho que hubiera significado un avance en el tratamiento didáctico del concepto, permitiendo al alumno iniciarse en conceptos de topología y ampliar sus conocimientos sobre la noción de límite.

\section{Análisis del bloque II (Resultados en tabla II)}

En el manual 3 se dice en la introducción «[...] ¿si $x$ se aproxima hacia $x_{\theta}$, los valores correspondientes de $f$ se aproximan hacia un número real $L$ o no? Si la respuesta es afirmativa, diremos que $L$ es el límite de $f$ cuando $x$ se

Tabla I

Elementos analizados en el estudio de los manuales del bloque I.

\begin{tabular}{|c|c|c|}
\hline BLOQUE I & $\begin{array}{l}\text { MANUAL núm.1 } \\
\text { (COU) }\end{array}$ & $\begin{array}{l}\text { MANUAL núm. } 2 \\
\left(1^{\circ} \text { de universidad) }\right.\end{array}$ \\
\hline 1. Introducción. & $\begin{array}{l}\text { A través de un ejemplo, usando la tabla de } \\
\text { valores y la gráfica de } f(x)=\frac{1 x^{2}-1}{4 x-1}\end{array}$ & $\begin{array}{l}\text { A través de un ejemplo, usando la tabla de } \\
\text { valores y la gráfica de } f(x)=\frac{1 x^{2}-1}{4 x-1}\end{array}$ \\
\hline 2. Definición. & Mérrica con entorno reducido simétrico. & Métrica con entorno reducido simétrico. \\
\hline 3. Ejemplos. & Sólo el ejemplo de introducción. & $\begin{array}{l}\text { Se dan tres ejemplos: } f(x)=K, f(x)=E(x) \text { y } \\
f(x)=x \operatorname{sen} l / x \text {. }\end{array}$ \\
\hline 4. Concepciones. & $\mathrm{CN}, \mathrm{CAM}$ & $\mathrm{CN}$, CAM. \\
\hline $\begin{array}{l}\text { 5a. Obstáculos } \\
\text { (OI) }\end{array}$ & $\mathrm{O}_{2}, \mathrm{O}_{3}$ & $\mathrm{O}_{2}, \mathrm{O}_{3}$ \\
\hline $\begin{array}{l}\text { 5b. Obstáculos } \\
\text { (ONS) }\end{array}$ & $\mathrm{O}_{2}, \mathrm{O}_{3}$ & $\mathrm{O}_{2}, \mathrm{O}_{3}$ \\
\hline 6. Dificuitades. & Mezclar la definición métrica y topológica. & Mezclar la definición métrica y topológica. \\
\hline
\end{tabular}


aproxima al número $x_{0}[\ldots] \gg$. Al no aclararse el término aproximación se induce a los obstáculos $\mathrm{O}_{3}$ y $\mathrm{O}_{4}$. Sólo al dar la definición métrica se dice, sin ninguna otra aclaración anterior o posterior, que «[...] el orden de aproximación de $x$ a $x_{0}$ se designa por $\delta$, luego $0<\left|x-x_{0}\right|<\delta$, ya que nunca puede tomar el valor de $x_{0},[\ldots] »$, y de forma análoga se define el orden de aproximación de $f(x)$ a $L$, todo ello sin acompañarse de un ejemplo, lo cual obliga al alumno a realizar un esfuerzo de abstracción muy superior para la edad y conocimientos del curso en que se encuentra. Posteriormente se dan otras definiciones (topológica, dominio de convergencia de la función, etc.), de todo lo cual se desprende, según el estudio de este manual, una excesiva formalización teórica y ausencia de ejemplos que motiven actos de comprensión para poder superar todos los obstáculos a los que se induce con esta presentación đel concepto. En el manual 4, la utilización de la gráfica de una función abstracta en la interpretación geométrica induce al obstáculo $\mathrm{O}_{2} \mathrm{y}$, al dar las definiciones métrica y topológica, se dice lo siguiente: «[...] si uno de los dos números $L$ o $x_{0}$, o ambos a la vez, se hacen infinito, la definición se expresa [...]». Con esta afirmación se favorece el obstáculo $\mathrm{O}_{6}$. No se facilitan ejemplos o situaciones que provoquen en los

Tabla II

Elementos analizados en el estudio de los manuales del bloque II.

\begin{tabular}{|c|c|c|c|c|}
\hline BLOQUE II & $\begin{array}{l}\text { MANUAL núm. } 3 \\
\text { (2 de BUP })\end{array}$ & $\begin{array}{l}\text { MANUAL núm. } 4 \\
\text { (COU) }\end{array}$ & $\begin{array}{l}\text { MANUAL núm. } 5 \\
\left(2^{\circ} \text { de BUP }\right)\end{array}$ & $\begin{array}{l}\text { MANUAL núm. } 6 \\
\text { (COU) }\end{array}$ \\
\hline 1. Introducción. & $\begin{array}{l}\text { Por analogía con límite } \\
\text { de sucesión y } 3 \text { ejem- } \\
\text { plos: } \mathrm{f}(\mathrm{x})=\mathrm{x}^{2} \\
g(x)=\frac{x^{2}-4}{x-2} \\
\mathrm{~h}(\mathrm{x})=\mathrm{E}(\mathrm{x})\end{array}$ & $\begin{array}{l}\text { A partir de la definición } \\
\text { topológica. }\end{array}$ & $\begin{array}{l}\text { A partir de la idea } \\
\text { intuitiva, con tabla de } \\
\text { valores y gráfica, ve los } \\
\text { límites laterales. }\end{array}$ & $\begin{array}{l}\text { A partir de la idea } \\
\text { intuitiva, con tabla de } \\
\text { valores y gráfica, ve } \\
\text { los límites laterales. }\end{array}$ \\
\hline 2. Definición. & $\begin{array}{l}\text { Definición métrica } \\
\text { informal. } \\
\text { Definición topológica. }\end{array}$ & $\begin{array}{l}\text { Por punto de } \\
\text { acumulación. } \\
\text { Definición métrica. }\end{array}$ & $\begin{array}{l}\text { Por sucesiones. } \\
\text { Definición métrica. }\end{array}$ & $\begin{array}{l}\text { Por sucesiones. } \\
\text { Definición métrica. }\end{array}$ \\
\hline 3. Ejemplos. & $\begin{array}{l}\text { Los tres ejemplos de la } \\
\text { introducción. }\end{array}$ & $\begin{array}{l}\text { La función } g \text { del ma- } \\
\text { nual } 3 \text {. }\end{array}$ & $\begin{array}{l}f(x)=x^{2}, g(x)=E(x) \\
h(x)=(x+I) / x\end{array}$ & $\begin{array}{l}f(x)=x^{2}, g(x)=E(x) \\
h(x)=(x+1) / x\end{array}$ \\
\hline 4. Concepciones. & $\mathrm{CN}, \mathrm{CAM}, \mathrm{CT}$. & CAM, CT. & $\mathrm{CN}, \mathrm{CAM}$ & CN, CAM. \\
\hline $\begin{array}{l}\text { 5a. Obstáculos } \\
\text { (OI) }\end{array}$ & $\mathrm{O}_{1}, \mathrm{O}_{2}, \mathrm{O}_{3}, \mathrm{O}_{4}, \mathrm{O}_{9}$ & $\mathrm{O}_{2}$ & $\mathrm{O}_{1}, \mathrm{O}_{2}, \mathrm{O}_{3}, \mathrm{O}_{4}, \mathrm{O}_{6}$ & $\mathrm{O}_{1}, \mathrm{O}_{2}, \mathrm{O}_{6}$ \\
\hline $\begin{array}{l}\text { 5b. Obstáculos } \\
\text { (ONS) }\end{array}$ & $\mathrm{O}_{1}, \mathrm{O}_{2}, \mathrm{O}_{3}, \mathrm{O}_{4}, \mathrm{O}_{9}$ & $\mathrm{O}_{2}$ & $\mathrm{O}_{1}, \mathrm{O}_{2}, \mathrm{O}_{3}, \mathrm{O}_{4}, \mathrm{O}_{6}$ & $\mathrm{O}_{1}, \mathrm{O}_{2}, \mathrm{O}_{6}$ \\
\hline 6. Dificulrades. & $\begin{array}{l}\text { La utilización de } \\
\text { funciones escalonadas } \\
\text { Ileva a un valor } \\
\text { constante de la } \\
\text { función. } \\
\text { Abuso de definiciones } \\
\text { informales. } \\
\text { Uso del valor } \\
\text { absoluto, sin haberlo } \\
\text { estudiado. } \\
\text { Utilización de } x_{0^{+}} x_{0^{-}} \\
\text {sin ejemplos. }\end{array}$ & $\begin{array}{l}\text { Cambio metodologico: } \\
\text { directamente la } \\
\text { definición. } \\
\text { Se apoya en la } \\
\text { definición topológica. } \\
\text { No se apoya en la } \\
\text { definición numérica. } \\
\text { Limites laterales sin } \\
\text { apoyo numérico ni } \\
\text { gráfico. } \\
\text { EI uso de funciones } \\
\text { trigonométricas en } \\
\text { primer lugar actúa de } \\
\text { distractor. }\end{array}$ & $\begin{array}{l}\text { El uso de funciones } \\
\text { escalonadas puede } \\
\text { hacer perder la idea } \\
\text { intuitiva de } \\
\text { aproximación. } \\
\text { Al no ser los límites } \\
\text { Iaterales iguales, «por } \\
\text { decreton, se dice que no } \\
\text { hay límite. } \\
\text { La definición métrica } \\
\text { actúa de distractor, l no } \\
\text { se relaciona con } \\
\text { ejemplos. }\end{array}$ & $\begin{array}{l}\text { No se ejercita el valor } \\
\text { absoluto, con lo que } \\
\text { los alumnos } \\
\text { encuentran una seria } \\
\text { dificultad en el } \\
\text { concepto de limite. } \\
\text { Tampoco se utiliza en } \\
\text { las propiedades. }\end{array}$ \\
\hline
\end{tabular}


alumnos actos de comprensión, por lo que se considera que este manual es de difícil seguimiento para los alumnos.

Respecto a los manuales 5 y 6 , de los mismos autores, cabe destacar que el enfoque con el que se presenta el concepto es completamente distinto al utilizado en la etapa anterior; del excesivo formalismo se pasa a un tratamiento más didáctico (se plantean en la introducción problemas de la vida real); no obstante, para determinados obstáculos, no se incluyen situaciones que permitan superarlos. Así, cuando se presenta la definición en la forma:

«Una función $f(x)$ tiene límite $L$ en el punto $x=u$, cuando para toda sucesión de valores $x_{n}$ que tenga por límite $u$, la sucesión de valores correspondientes $f\left(x_{n}\right)$ tiene por límite $L$. Se indica así:

$$
\begin{gathered}
\lim _{x \rightarrow a} f(x)=L \\
\left.\left.\mathrm{u}=\mathrm{a}, \mathrm{a}^{-}, \mathrm{a}^{+},+\infty, \infty-\quad \mathrm{L}=\mathbf{R},+\infty,-\infty\right)\right\rangle
\end{gathered}
$$

se induce al alumno a creer que las variables independiente y dependiente toman el valor $+\infty y-\infty$, (obstáculo $\mathrm{O}_{6}$ ). Otro hecho a destacar es que se repiten en ambos manuales la introducción, los ejemplos y definición formal del concepto de limite de una función, señalando que en el manual 6 se ha omitido de la definición $\ll \mathrm{u}=\mathrm{a}$, $a^{-}, a^{+},+\infty,-\infty$ y $L=R,+\infty,-\infty \gg ;$ por lo cual ya no induce a obstáculo y se puede afirmar que derivan las mismas concepciones. Los autores distinguen los dos cursos a los que se dirigen los manuales 5 y 6 mediante la inclusión de los ejemplos

$$
\lim _{x \rightarrow 0} \frac{\operatorname{sen} x}{x}
$$

y

$$
\lim _{x \rightarrow 0} \frac{1-\cos x}{x}
$$

\begin{tabular}{|c|c|c|c|}
\hline BLOQUE III & $\begin{array}{l}\text { MANUAL núm. } 7 \\
\left(2^{\circ} \text { de BUP }\right)\end{array}$ & $\begin{array}{l}\text { MANUAL núm. } 7 \\
\text { (COU) }\end{array}$ & $\begin{array}{l}\text { MANUAL núm. } 8 \\
\text { (1 }{ }^{\circ} \text { de universidad) }\end{array}$ \\
\hline l. Introducción. & $\begin{array}{l}\text { A través de un ejemplo de la } \\
\text { vida real para el límite en el } \\
\text { infinito }\end{array}$ & $\begin{array}{l}\text { Por límites laterales se llega al } \\
\text { concepto de límite. }\end{array}$ & $\begin{array}{l}\text { Con la definición mediante } \\
\text { límite de una sucesión } \\
\text { Luego dan los límites } \\
\text { laterales. }\end{array}$ \\
\hline 2. Definición. & Definición métrica. & $\begin{array}{l}\text { Definiciones métricas de los } \\
\text { límites laterales. }\end{array}$ & $\begin{array}{l}\text { Mediante sucesiones. } \\
\text { Definición métrica. }\end{array}$ \\
\hline 3. Ejemplos. & $\begin{array}{l}\text { Ejemplo con } f(x)=x^{2} \text {, que se } \\
\text { liga a la continuidad } \\
\text { Otros ejemplos excesivamente } \\
\text { complicados. }\end{array}$ & Ninguno. & $\begin{array}{l}-\mathrm{f}(\mathrm{x})=1 / \mathrm{x} \\
-\mathrm{f}(\mathrm{x})=0 \text { si } x \text { es irracional, } \mathrm{y} \\
\mathrm{f}(\mathrm{x})=1 / \mathrm{q} \text { si } x \text { es racional } \\
-\mathrm{funcion} \text { de Dirichlet. } \\
-\mathrm{f}(\mathrm{x})=\mathrm{x} / \operatorname{sen} \mathrm{x} \\
- \text { otros. }\end{array}$ \\
\hline 4. Concepciones. & CAM. & CAM. & $\mathrm{CN}, \mathrm{CAM}$ \\
\hline $\begin{array}{l}\text { 5a. Obstáculos } \\
\text { (OI) }\end{array}$ & $\mathrm{O}_{2}, \mathrm{O}_{3}, \mathrm{O}_{4}, \mathrm{O}_{6}, \mathrm{O}_{7}, \mathrm{O}_{9}$ & $\mathrm{O}_{2}, \mathrm{O}_{6}$ & $\mathrm{O}_{2}, \mathrm{O}_{3}, \mathrm{O}_{6}, \mathrm{O}_{7}, \mathrm{O}_{9}$ \\
\hline $\begin{array}{l}\text { 5b. Obstáculos } \\
\text { (ONS) }\end{array}$ & $\mathrm{O}_{2}, \mathrm{O}_{3}, \mathrm{O}_{4}, \mathrm{O}_{6}, \mathrm{O}_{7}, \mathrm{O}_{9}$ & $\mathrm{O}_{2}$ & \\
\hline 6. Dificultades. & $\begin{array}{l}\text { No se ejercita el valor } \\
\text { absoluto, con lo que los } \\
\text { alumnos encuentran una seria } \\
\text { diffcultad en el concepto de } \\
\text { limite. }\end{array}$ & $\begin{array}{l}\text { No se ejercita el valor } \\
\text { absoluto, con lo que los } \\
\text { alumpos encuentran una seria } \\
\text { dificuttad en el concepto de } \\
\text { limite. }\end{array}$ & $\begin{array}{l}\text { No se usa la representación } \\
\text { grafica ni la tabla de valores } \\
\text { de las funciones como ayuda } \\
\text { didáctica aunque se indica su } \\
\text { utilidad. }\end{array}$ \\
\hline
\end{tabular}

y, por último, se definen funciones equivalentes.

Tabla III

Elementos analizados en el estudio de los manuales del bloque III. 


\section{Análisis del bloque III (Resultados en tabla III)}

En el manual 7 están presentes los obstáculos $\mathrm{O}_{2}, \mathrm{O}_{3}, \mathrm{O}_{4}$, $\mathrm{O}_{6}, \mathrm{O}_{7}, \mathrm{O}_{9}$, expresiones como las que se señalan a continuación y que aparecen en el texto justifican su existencia en el mismo; así, se induce al obstáculo $\mathrm{O}_{2}$ cuando, al plantearse como ejemplo medir la amplitud de unas oscilaciones, se presenta una gráfica y se dice «la gráfica, en verde, es la curva a la que se aproximan los datos obtenidos por nuestras amigas. ¿Te animas a seguir la experiencia? Si lo haces podrás observar que

$$
\lim _{x \rightarrow \infty}
$$

Al no plantearse situaciones que puedan provocar en el alumno alguno de los actos de comprensión que permitan superar los anteriores, dichos obstáculos aparecen como (ONS). En el manual 8 se detecta la presencia de los obstáculos $\mathrm{O}_{2}$ y $\mathrm{O}_{6}$, ya que se introduce el concepto y se da la definición utilizando la gráfica de una función abstracta; sin embargo, se provoca en el alumno actos de comprensión que le permiten la superación de $\mathrm{O}_{6}$ cuan-

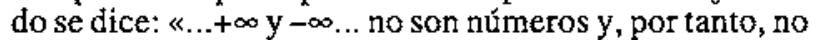
se pueden operar ni comparar con los números».

Respecto al manual 9, en él se ha observado un estudio muy completo de todos los aspectos que llevan a la comprensión del concepto de límite de una función en un punto; se plantean multitud de situaciones que facilitan actos de comprensión una vez dada la definición de limite mediante sucesiones, por to que se describen a continuación algunas de ellas:

$-L$ puede ser un número y también $+\infty$ y $-\infty$. (Sería un acto de comprensión para $\mathrm{O}_{6}$.)

- Cuando se trata de investigar si existe el límite de $f$ cuando $x$ tiende a $c$, conviene empezar eligiendo una sucesión de números distintos de $c .$. . (Sería un acto de comprensión para $\mathrm{O}_{9}$.)

- En algunos casos el límite de una función se puede obtener muy fácilmente. Por ejemplo, $5 x, 8 x$ y $7 x^{2}$ tienen,

Elementos analizados en el estudio de los manuales del bloque IV.

\begin{tabular}{|c|c|c|}
\hline BLOQUE IV & $\begin{array}{l}\text { MANUAL núm.10 } \\
\left(1^{\circ} \text { de universidad }\right)\end{array}$ & $\begin{array}{l}\text { MANUAL núm. } 11 \\
\left(1^{\circ} \text { de universidad }\right)\end{array}$ \\
\hline 1. Introducción. & $\begin{array}{l}\text { Con la ayuda de la gráfica de } 2 \text { funciones } \\
\text { abstractas. } \\
\text { Definición informal (aproximación numérica). }\end{array}$ & $\begin{array}{l}\text { Apoyándose en eí límite de una surcesión. } \\
\text { Luego dan los límites laterales. }\end{array}$ \\
\hline 2. Definición. & $\begin{array}{l}\text { Métrica. } \\
\text { Topológica. }\end{array}$ & $\begin{array}{l}\text { A través de la de sucesión. } \\
\text { Da la def. métrica a través de un teorema. }\end{array}$ \\
\hline 3. Ejemplos. & $\begin{array}{l}f(x)=E(x) ; f(x)=K ; f(x)=x ; f(x)=x \text { sen } 1 / x \text { y } \\
\text { funciones polin. y rac. de } 2^{\circ} \text { grado. }\end{array}$ & $\begin{array}{l}\mathrm{f}(\mathrm{x})=1 / \mathrm{x} ; \\
\mathrm{f}(\mathrm{x})=0 \text { si } x \text { es irracional, } \mathrm{y}(\mathrm{x})=1 / \mathrm{q} \text { si } x \text { es } \\
\text { racional. } \\
\text { función de Dirichlet; } \mathrm{f}(\mathrm{x})=\mathrm{x} / \operatorname{sen} \mathrm{x} \text { y otros. }\end{array}$ \\
\hline 4. Concepciones. & $\mathrm{CN}, \mathrm{CAM}, \mathrm{CT}$ & $\mathrm{CN}, \mathrm{CAM}$ \\
\hline $\begin{array}{l}\text { 5a. Obstáculos } \\
\text { (OI) }\end{array}$ & $\mathrm{O}_{2}, \mathrm{O}_{3}, \mathrm{O}_{4}, \mathrm{O}_{5}$ & $\mathrm{O}_{2}, \mathrm{O}_{3}, \mathrm{O}_{6}, \mathrm{O}_{7}, \mathrm{O}_{9}$ \\
\hline $\begin{array}{l}\text { Sb. Obstáculos } \\
\text { (ONS) }\end{array}$ & $\mathrm{O}_{2}, \mathrm{O}_{3}, \mathrm{O}_{4}, \mathrm{O}_{5}$ & \\
\hline 6. Dificultades. & $\begin{array}{l}\text { La secuenciación de ejemplos no es correcta al } \\
\text { no graduarse progresivamente la dificultad } \\
\text { éstos. } \\
\text { Planteamiento con ausencia de una } \\
\text { aproximación numérica que hace demasiado } \\
\text { abstracto el concepto. }\end{array}$ & $\begin{array}{l}\text { No se usa la representación gráfica ni la tabła de } \\
\text { valores de las funciones como ayuda didáctica, } \\
\text { aunque se indica su utilidad. }\end{array}$ \\
\hline
\end{tabular}


las tres, límite 0 cuando $x$ tiende a $0 .$. La forma de tender a 0 es distinta para cada una de ellas y esto se puede poner de manifiesto considerando los cocientes entre ellas..., y una vez justificado, el autor indica que $7 x^{2}$ se aproxima a 0 más rápidamente. (Sería un acto de comprensión para $\mathrm{O}_{3}$.)

- Para «cada» $\varepsilon$ positivo hay que hallar $r$ tal que... es menor que $\varepsilon$. (Sería un acto de comprensión para $\mathrm{O}_{7}$.)

- Posteriormente plantea el problema de cómo cuantificar la mayor o menor aproximación de $x$ a $c$ y de $f(x)$ a $L$. (Sería otro acto de comprensión para $\mathrm{O}_{3}$.)

Por todo lo anterior se desprende, como hecho destacable en este bloque, que en el manual 9 , destinado a la enseñanza universitaria, el concepto de limite de una función tiene un mejor tratamiento didáctico que en los de enseñanza media (manuales 7 y 8); ya que, por una parte, en el manual 9 se apoyan en la idea de límite de una sucesión tratando de conectar con las ideas previas de los estudiantes; $y$, por otra, se plantean ejemplos que permiten que los alumnos superen gran número de obstáculos inherentes al concepto, por lo que éste se ve enriquecido, mientras que en los otros dos manuales se utilizan sólo las definiciones sin facilitar que se provoquen actos de comprensión.

\section{Análisis del bloque IV (Resultados en tabla IV)}

En este bloque nos referiremos básicamente al manual 10 , ya que el manual 11 es el llamado manual 9 en el bloque III. En la introducción al tema se indica que, a partir de la definición de función real y del estudio de algunas representaciones gráficas, se puede llegar al concepto de límite de forma intuitiva. Posteriormente, en el apartado límites, considera las gráficas de dos funciones $f$ y $g$ iguales, excepto en $x=x_{0}$, para el que $f$ no está definida y $g$ toma el valor 3 , indicando, a partir de la gráficas, que el límite de ambas funciones, cuando $x$ tiende a $x_{0}$, es 3 ; de esta manera se induce al alumno al obstáculo $\mathrm{O}_{2}$, ya que no se plantea ningún ejemplo en el que no sea posible determinar la existencia de límite con Ia sola observación de la gráfica (p.e., $f(x)=\operatorname{sen} 1 / x$, cuando $x$ tiende a 0 ). Más adelante se dice: «Por pequeño que sea $\varepsilon>0 \ldots$ existe un $\delta>0 \ldots$, sólo tenemos que observar la figura para darnos cuenta de que existe $\delta . .$. con ello se induce al obstáculo $\mathrm{O}_{7}$.

Aparecen diferencias acusadas entre los manuales de este bloque. Mientras los autores del manual 10 siguen un discurso con una clara tendencia hacia la demostración analítica, sin preocuparse de las aproximaciones numéricas, en el manual 11 se da un enfoque didáctico en el que, en la primera parte, la aproximación numérica es el centro de atención, realizándose una conexión adecuada con la vía analítica.

Se observa, en ambos manuales, una ausencia de representaciones gráficas que podrían apoyar los razonamientos que se efectúan en los mismos; a pesar de ello, en el manual 11 se indica expresamente la utilidad de las gráficas.

\section{CONCLUSIONES}

El análisis de manuales efectuado permite hacer, en función de cada una de las variables estudiadas, numerosas consideraciones, entre las que se destacan las siguientes:

Según la forma de introducir la noción, se detecta que, desde el año 1990, los autores tienden a tener en cuenta los conocimientos previos del alumno, apoyándose en el concepto de límite de una sucesión, lo cual indica una evolución positiva en este sentido.

Respecto a la definición, se observa en los últimos años una tendencia que se considera también positiva en la evolución del tratamiento del concepto: prescindir de la definición topológica de la noción de limite de una función en tex tos de enseñanza no universitaria, cuestión que era considerada con cierto rigor en manuales dirigidos a alumnos de enseñanza media en el período 1983 . 1987.

Con relación a los ejemplos que se presentan, son reite* rativos en los distintos niveles, las funciones dadas por

$$
f(x)=x^{2}, f(x)=E(x) \text { y } f(x)=\frac{x^{2}-4}{x-2}
$$

aparecen en la mayoría de los manuales, sea cual sea el nivel de enseñanza al que está orientado; en el caso de usar otros ejemplos, se suelen presentar confusamente, como en el caso del manual 7, exceptuando los que se incluyen en el manual 9, que abarcan una gran cantidad de situaciones respecto de la noción de limite de una función.

En cuanto a las concepciones, se ha detectado la existencia de épocas en las que el tratamiento dado al estudio del límite de una función en un punto tiene más rigor en ciertos manuales de nivel de enseñanza no universitaria que en los manuales de enseñanza universitaria de otra época distinta. Así, de los manuales 3 y 4 (correspondientes a $2^{\circ}$ de BUP y COU), se derivan las concepciones CN, CAM y CT y, sin embargo, la CT no se extrae del manual 9 (correspondiente a $1^{\circ}$ de universidad). Un hecho relevante es la ausencia casi generalizada de situaciones didácticas en las que aparezca la concepción geométrica de límite.

Respecto a las dificultades y obstáculos que se observan en los manuales, en la mayor parte de los textos analiza. dos se han detectado situaciones que pueden provocar obstáculos en el alumno. Algunos de ellos son epistemológicos, se deben a la propia noción, y otros, como por ejemplo el $\mathrm{O}_{2}, \mathrm{O}_{6}$ y el $\mathrm{O}_{9}$, son debidos a la propia transposición didáctica del saber; son, por tanto, didácticos. En la mayoría no se incluyen, tanto para los obstáculos epistemológicos como para los didácticos, situaciones que provoquen en los alumnos los actos de comprensión necesarios. Sin embargo, se observa que el tratamiento didáctico que recibe la noción de límite ha evolucionado positivamente, ya que en los mantuales 
más actuales, al favorecer situaciones que provoquen en el alumno actos de comprensión, se ayuda a superar todos los obstáculos. Entre las dificultades observadas destacan: el uso del valor absoluto en manuales de segundo de BUP, suponiéndolo conocido, lo cual no tiene por qué ser cierto $y$, sin embargo, en otros manuales de COU no se ejercita al alumno en dicho concepto; el uso abusivo de definiciones informales y la utilización de términos como $x_{0}{ }^{+}$y $x_{0}-$ sin el apoyo de ejemplos aclaratorios y mezclar las definiciones métrica y topológica. La evolución de los conceptos, a través de los distintos niveles y para un mismo autor, no aparece como tal, al dar a la noción de límite de una función el mismo tratamiento en manuales de distinto nivel; por lo que se manifiestan las mismas concepciones y obstáculos a pesar de estar destinados a alumnos con un mayor desarrollo intelectual, hecho que no favorece la evolución conceptual en los alumnos. En algunos manuales se realizan transposiciones didácticas no adecuadas, ya que para algunos autores el hecho de disponer de un ejemplo que sea de la «vida real» justifica su inclusión en el texto aunque dé Iugar a la aparición de obstáculos didácticos y no se facilite su superación.

\section{REFERENCIAS BIBLIOGRÁFICAS}

BACHELLARD, G. (1983). Laformación delespiritu científico. Buenos Aires: Siglo XXI.

CHEVALLARD, 1. y JOHSUA, M.A. (1991). La transposition didactique. Grenoble: La Pensée Sauvage.

CORNU, B. (1983). Apprentissage de la notion de limite. These de doctorat de troisième cycle de Mathématiques pures. Université de Grenoble.

DELEDICQ, A. (1994). Les conceptions relatives aux limites. Vingt ans de didactique des mathématiques en France. $M$. Artigue, R. Grass, C. Laborde y P. Tavignot (eds.), pp. 321 327. Grenoble: La Pensée Sauvage.

EL BOUAZZAOUI, H. (1988). Conceptions des élèves et des professeurs a propos de la notion de continuité d'une fonction. PHD. Université de Bordeaux I.

HENRY, M. (1991). Une presentation de la didactique en vue de la formation des enseignants. IREM. Besançon.

RICO, L. (1992). Investigación sobre errores de aprendizaje en educación matemática. Proyecto de investigación presentado a concurso-oposición de catedráticos de Universidad de Didáctica de la Matemática en la Universidad de Granada.

ROMERO MARTÍNEZ, L. (1991). Un modelo didactico para la adquisición del concepto de límite. Tesis doctoral. Universidad de Murcia.

SÁNCHEZ, C. y CONTRERAS, A. (1995a). Concepciones de los alumnos de COU en torno a la noción de limite de una
Por todo lo anterior, se considera necesario que, en un libro de texto dirigido a estudiantes, se presente el concepto de límite de una función de modo que inchya un abanico de situaciones didácticas, lo más amplio posible, que favorezcan en el alumno los actos de comprensión que le permitan superar el mayor número de obstáculos. Con ello, la comprensión del concepto se facilitaría y se vería enriquecida. Ello trae consigo multitud de consecuencias, señalando que, como pauta general, es importante que la introducción del concepto se realice a través de un ejemplo, con la triple representación gráfica, numérica y analítica y que, en ese primer ejemplo que se utilice, la función considerada no sea continua en el punto en el que se calcula el límite o éste no sea del dominio de la función; de esta forma se consigue en la presentación del concepto inducir y facilitar la superación de un gran número de obstáculos. Se considera fundamental que haya una verdadera evolución del concepto en cuanto a los niveles académicos, tratando de desarrollarlo progresivamente según la complejidad del mismo, lo cual se facilitaría si un mismo equipo redactase los textos dirigidos a los cursos que enlazan la enseñanza en esos sucesivos niveles.

función. VII Jornadas Andaluzas de Educación Matemática «THALES». Córdoba.

SÁNCHEZ, C. y CONTRERAS A (1995b). Epistemología deI concepto de límite. Análisis de manuales. VIÎ JAEM. Madrid.

SÁNCHEZ, C. y CONTRERAS, A. (1996a). Esiudio del proceso de enseñanza-aprendizaje del concepto de límite de una función en diplomaturas de escuelas técnicas. ICME, 8. Sevilla.

SCHUBRING, G. (1985). Essais surl'histoire de l'enseignement des Mathématiques. Recherches en Didactique des Mathématiques, Vol. 5(3).

SCHUBRING, G. (1987). On the Methodology of Analysing Historical Textbooks: Lacroix as Textbooks Author. For the Learning of Mathematics, Vol. 7(3).

SIERPINSKA, A. (1985). Obstacles épistémologiques relatifs a la notion de limite. Recherches en Didactique des Mathématiques, Vol. 6(1).

SIERPINSKA, A. (199I). Some remarks on understanding in mathematics. Version revisada del trabajo presentado al Canadian Mathematics Education Study Group. Vancouver.

TALL, D. (1995). Seminariode Didáctica del Análisis y Didáctica de las Funciones. Universidad Autónoma de Barcelona.

WEBER, J. (1986). Basic content analysis. Newbury Park, California: Sage University Press. 


\section{MANUALES ANALIZADOS}

Manual 1. RÍOS, S. (1974). Matemáticas especiales (COU), Madrid: Editorial Paraninfo.

Manual 2. RÍOS, S. (1973). Cálculo infinitesimal. Madrid: Editorial Paraninfo.

Manual 3. VIZMANOS, J.R., ANZOLA, M. y PRIMO, A. (1983). Matemáticas 2 BUP. Madrid: Ediciones SM.

Manual 4. PRIMO, A. (1987). Matemáticas COU. Madrid: Ediciones SM.

Manual 5. VIZMANOS, J.R. y ANZOLA, M. (1990). Matemáticas-Algoritmo 2 BUP. Madrid: Ediciones SM.
Manual 6. VIZMANOS, J.R y ANZOLA, M. (1993). Matemáticas I. COU. (Opción A-B). Madrid: Ediciones SM.

Manual 7. GUZMÁN, M., COLERA, J. y SALVADOR, A. (1987). Matemáticas. Bachillerato 2. Navarra: Ediciones Anaya.

Manual 8. GUZMÁN, M. y COLERA, I. (1989). Matemáticas I. COU. Navarra: Ediciones Anaya.

Manual 9, 11. GUZMÁN, M. y RUBIO, B. (1992). Problemas, conceptosymétodos del análisis matemático. Madrid: Pirámide.

Manual 10. GARCÍA CASTRO, F. y GUTTÉRREZ GÓMEZ, A. (1992). Cálculo infinitesimal I, Vol. 1. Madrid: Pirámide.

[Artículo recibido en enero de 1996 y aceptado en junio de 1997.] 